\title{
Das geplante «Datenschutz-Gesetz» - eine Bedrohung der medizinisch-epidemiologischen Forschung in der Schweiz?
}

\author{
Ursula Ackermann-Liebrich, R. Bruppacher, F. Gutzwiller, Ch. Minder, H. Noack, F. Paccaud, G. Schüler, \\ B. Somaini, R. Steffen, P. Wüthrich
}

\begin{abstract}
Uns allen ist der Schutz persönlicher Information vor Missbräuchen vordringlich: ein Bundesgesetz über den Schutz von Personendaten ("Datenschutz-Gesetz") ist notwendig und uberfällig. Aber ein solches Gesetz darf den Schutz der Bevölkerung vor gesundheitlichen Gefahren nicht verunmöglichen.
\end{abstract}

Gemäss vorliegendem Entwurf bestimmt das Datenschutzgesetz:

- Jede nicht gesetzlich vorgeschriebene Bearbeitung von persönlichen Daten durch die Bundesverwaltung ist rechtswidrig; die Datenbearbeitung zur Analyse, Veröffentlichung etc. durch Private (auch Wissenschaftler, Universitats- und andere Institute etc.) wird stark eingeschränkt.

Die Folgen sind:

- Die Entdeckung, Abklärung und Verhitung von neu auftretenden, für die Bevölkerung bedrohlichen gesundheitlichen Entwicklungen (Beispiele: Epidemien, AIDS, Nahrungsmittelvergiftungen, gesundheitsschädigende Substanzen in Medikamenten, Folgen der Umweltverschmutzung, Gesundheitsrisiken am Arbeitsplatz) wird erschwert oder gar verunmöglicht.

- Die Entscheidungen über die Durchführung epidemiologischer, medizinischer und gesundheitsökonomischer Untersuchungen werden nicht mehr von wissenschaftlichen Experten sondern von der Datenschutzkommission gefallt. Beamte des Datenschutzes werden z.B. festlegen, welche epidemiologischen untersuchungen im öffentlichen Interesse liegen.

- Die zu schaffende Datenschutz-Verwaltung wird Umtriebe, Verzögerungen und Kosten für
Staat, medizinische und epidemiologische Forschung verursachen.

Diese Folgen sind unsres Erachtens vom Standpunkt der Volksgesundheit her nicht tragbar. Deshalb fordern wir einen gesetzlichen Rahmen, der eine flexible, differenzierte und transparente Regelung des Datenschutzes in der epidemiologischen und medizinischen Forschung erlaubt.

\section{Summary}

Implementations of the new law on the confidentiality of personal data

The draft of the new law on the confidentiality of personal data severely curtails medical and epidemiological research. This might be detrimental and dangerous to public health. The project therefore has to be amended.

Résume

Les conséguences de la nouvelle loi pour la protection des données

La nouvelle loi pour la protection des donnees limite la recherche médicale, en particulier dans le domaine de l'épidémiologie. Une menace pour la sante de la population pourrait en résulter. Des modifications correspondantes sont indispensables.

\section{Korrespondenz an}

Dr. Ch. Minder, Institut für Sozial- und Prăventivmedizin der Universität Bern, Finkenhubelweg 11, 3012 Bern. 